\title{
Inhaltsverzeichnis
}

\author{
(Anschließend das Gesetzesregister)
}

\section{Die Fälle}

(S. 3-36 des Heftes in der Schlaufe des hinteren Deckels)

\section{Die Lösungen zum Ersten Teil der Fälle}

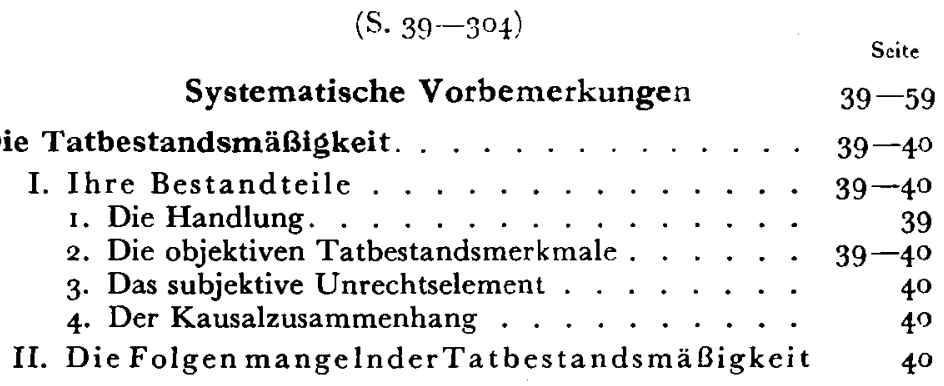

A. Die Tatbestandsmäßigkeit. . . . . . . . . . . . . 39-40

B. Die Rechtswidrigkeit. . . . . . . . . . . . . . . . $40-43$

I. Die Regel. . . . . . . . . . . . . . . $44^{\circ}$

II. Die objektive Rechtswidrigkeit ist kein Tatbestandsmerkmal ........... . $44^{I}$

III. Rechtswidrigkeitsausschließungsgründe $\cdot 4^{1-43}$

IV. Die Folgen mangelnder Rechtswidrigkeit . 43

C. Die Schuld . . . . . . . . . . . . . . . . $42-58$

I. Das Wesen der Schuld . . . . . . . . 43

II. DiebiologischenVoraussetzungender Schuldbejahung . . . . . . . . . . . . . . . . 44-45

I. Die altersmäßige Verstandes- und Willensreife . . 44

2. Die Zurechnungsfähigkeit . . . . . . . . 44-45

III. Die Schuldformen .. . . . . . . $45-46$

I. Der direkte oder unbedingte Vorsatz . . . . . . 45

2. Der bedingte Vorsatz (dolus eventualis) . . . 45-46

3. Der dolus generalis . . . . . . . . . . . . 46

4. Die Fahrlässigkeit . . . . . . . . . . . . . . $44^{6}$

IV. Die Schuldausschließungsgründe . . . . . $4^{6-4^{8}}$

1. Die Schuldausschließungsgründe i. e. S. . . . . 46

2. Entschuldigungsgründe .. . . . . . . $4^{6-4^{8}}$ 
V. Die Folgen eines Schuldausschließungsgrundes... . . . . . . . . . . . . . . $4^{8}$

VI. Tatbestands-u. Verbotsirrtum insbesondere. $48-55$

I. Der Tatbestandsirrtum . . . . . . . . . . . . 49-50

2. Der Verbotsirrtum . . . . . . . . . . . $59-55$

VII. Besondere, für die Schuldfrage bedeutungslose Irrtumsfälle . . . . . . . . . . .

I. Objektverwechslung . . . . . . . . . . . . .

2. Irrtum über Kausalverlauf . . . . . . . . . . . .

3. Wahnverbrechen . . . . . . . . . . . . . .

VIII. Bedingungen der Strafbarkeit und Verfolgbarkeit................ I. Die Regel . .

2. Bedingungen der Strafbarkeit . . . . . . .

3. Bedingungen der Verfolgbarkeit . . . . . . . .

D. Die persönlichen strafbefreienden Gründe . . . . . $5^{8-59}$

I. Strafausschließungsgründe . . . . . . 58

II. Strafaufhebungsgründe. . . . . . . . . . . 59

III. Die Folgen der persönlichen strafbefreienden Gründe . . . . . . . . . . . . . . 59

1. Fall: Ein unerfreulicher Bräutigam . . . . . . . 3

Lösung . . . . . . . . . . . . . . . 60-88

Allgemeiner Teil: Straflose Nachtat (Verwertungsdelikt gegenüber Aneignungsdelikt) S. 65. Der Strafantrag S. 77 und 86-88. Deliktscharakter der ,besonders schweren Fälle" S. 80.

Besonderer Teil: Betrug (durch Vorenthalten der Wahrheit) S. 6o. Unterschlagung (durch Ableugnen des Besitzes) S. 6ı. Tateinheit zwischen Unterschlagung und Betrug S. 63. Vermögensschädigung beim Betrug S. 64. Tatbestandsirrtum bei Unterschlagung S. 64. Kreditbetrug (Vorspiegelung falscher Tatsachen) S. 66. Unterschlagurig bei Sicherungsübereignung S. 68. Versuchte Unterschlagung S. 69. Betrug durch Übereignung einer fremden Sache S. 69 . Betrug durch Zahlung mit ungedecktem Scheck S. 7o und 72. Kausalität zwischen Irrtum und Vermögensverfügung $S .71$. Betrug durch abredewidrige Ausfüllung eines Blankoakzepts S. 72. Kein gegen Betrug ungeschütztes Vermögen S. 74. Betrug durch Wechselverfälschung S.76. Eingehungsbetrug und Erfüllungsbetrug (Erschleichung von Dienstverträgen) S. 78. 
Rückfallvoraussetzungen beim Betrug S. 79. Persönliche Begünstigung bei Strafantragsdelikten S. 80 . $\S \S 43,59,246,263$; ferner: Art. 10, 17 und 69 des Wechselgesetzes.

Ferner: Betrugstatbestände außerhalb des $\begin{array}{lll}\S & 263\end{array}$ (insbesondere Versicherungsbetrug und Automatenmißbrauch) S. 8I ff. Unterschlagungstatbestände außerhalb des $\S 246$ (Depotunterschlagung) S. $84 \mathrm{fr}$.

2. Fall: Der Nachschlüsseldieb und sein Gehilfe. . .

Lösung . . . . . . . . . . . . . . . . . . .

Allgemeiner Teil: Die Teilnahme (allgemeine Erörterungen zu $\S 50$ Abs. I und 2 mit Beispielen) S. 9r ff. Die Beihilfe (allgemeine Erörterungen S. 96-I I ; der vorliegende Fall S. IO I). Versuch, Rücktritt und tätige Reue (allgemeine Erörterungen mit Beispielen S. I I3-I I8; der vorliegende Fall, insbesondere Rücktritt des Teilnehmers S. 103-105).

Ferner: Fortgesetzte Tat und Kollektivdelikte (allgemeine Erörterungen) S. I $8-123$.

Besonderer Teil: Rückfalldiebstahl S. 89. Betrug durch Verkauf gestohlener Sachen S. 9o. Begünstigung (allgemeine Erörterungen S. 105--108; der vorliegende Fall S. ro8-I I I, insbesondere Selbstbegünstigung S. I Io und Begünstigung als Beihilfe S. I I I). Hehlerei (an erschwindeltem Geld S. I I I an umgearbeiteter Sache S. I12).

$\S \S 43,46,47,48,49,50,240,243$ I $3,244,245,257$, $258,259,263,319$ Z. I.

3. Fall: Der falsche Kriminalbeamte . . . . . . . . . 7

Lösung . . . . . . . . . . . . . . . 123-148

Allgemeiner Teil: Anstiftung zur Begünstigung bezüglich der eigenen Tat S. I 3 I.

Besonderer Teil: Amtsanmaßung (allgemeine Erörterungen S. I23-125; der vorliegende Fall S. 125). Hausfriedensbruch S. 125. Nötigung S. 125. Urkundenfälschung (öffentliche Urkunde S. I26; Mißbrauch von Ausweispapieren S. 127). Diebstahl eines Sparkassenbuchs (allgemeine Erörterungen zum Problem der Zueignungsabsicht S. 128-130; der vorliegende Fall S. I 30). Sachliche Begünstigung (Unterschied gegenüber Sachhehlerei S. 133-134; der vorliegende Fall S. 134-135).

$\$ \$ 48,123,240,242,257,25^{8}, 259,263,267,28$ I. 
Ferner: Allgemeine Diebstahlserörterungen (einfacher Diebstahl S. 136-14I; schwerer Diebstahl S. I4I - I 45; Rückfalldiebstahl S. I45-I 46 ; Besitz von Diebeswerkzeug S. I46-147; Spezialfälle des Diebstahls S. 147-148).

4. Fall: Irrtum kann vor Strafe schützen . . . . . . 8 Lösung . . . . . . . . . . . . . . $148-156$ Allgemeiner Teil: Tatbestandsirrtum S. I 54; Verbotsirrtum S. I55. Beihilfe gegenüber einem im Irrtum befindlichen Täter S. $155-156$.

Besonderer Teil: Vollstreckungsvereitelung (allgemeine Erörterungen S. I48-I 50; der vorliegende Fall S. 150-151). Verstrickungsbruch (allgemeine Erörterungen S. $151-153$; der vorliegende Fall $S$. I 53 - I 56 ).

$\$ \S 49,59$, I $37,288$.

5. Fall: Der gewissenlose Rechtsagent . . . . . . 99 9

Lösung . . . . . . . . . . . . . . . . I57-194

Allgemeiner Teil: Untersagung der Berufsausübung S. 18o. Eventualdolus bei Hehlerei S. 188 .

Besonderer Teil: Mittelbare (intellektuelle) Urkundenfälschung (Begriff der öffentlichen Urkunde) S. 157. Meineid (allgemeine Erörterungen zu $\$ \S I_{53}-163 \mathrm{~S}$. 157-I6I; der vorliegende Fall S. 16I). Prozeßbetrug (allgemeine Erörterungen S. 162 -164 ; der vorliegende Fall S. I64). Untreue (allgemeine Erörterungen S. I64-I68; der vorliegende Fall S. I68-1 70). Betrug durch Bewirkung eines Verzichts auf eine nichtige Forderung S. I 7 r. Urkundenfälschung (allgemeine Erörterungen S. I72-I 79; insbesondere Herstellung einer unechten Urkunde S. 176-1 79; der vorliegende, Fall S. I79-180). Sachhehlerei (allgemeine Erörterungen S. I8I-I88; der vorliegende Fall S. $188-189)$.

$\$ \S$ I53ff., 246, 259, 263, 266, 267, 27 I.

Ferner: Untreuetatbestände außerhalb des $\S 266$, S. 189-1 ro. Hehlereitatbestände außerhalb des $\S 259$, S. I9o. Die Wahlfeststellung, S. 190-192. Fälschung von Gesundheitszeugnissen ( $\$ 277-279)$ S. 192-194.

6. Fall: Der Münzfälscher Johannes Steidel . . . . . Io Lösung . . . . . . . . . . . . . . . . I 194-218

Allgemeiner Teil: Gesetzeskonkurrenz (allgemeine Erörterungen S. 198-200; der vorliegende Fall S. 
200-202). Tateinheit und Tatmehrheit (allgemeine Erörterungen S. 213-215; der vorliegende Fall S. 206-208). Mittäterschaft bei zweiaktigen Straftaten (allgemeine Erörterungen S. 2II-2I2; der vorlieliegende Fall S. 212-2I3).

Besonderer Teil: Münzverbrechen und Münzvergehen (allgemeine Erörterungen) S. 194-197. Abschieben von Falschgeld S. 197; im Zusammentreffen mit Betrug S. 200-202. Versuchtes Münzverbrechen S. 203. Strafbare Vorbereitungshandlung S. 203-205. Vollendete Falschmünzerei S. 205; im Zusammentreffen mit Betrug S. 206-209. Unterschlagung (an Fundsache S. 202; zum Nachteil eines Mittäters S. 205-206). Verbreitung von Falschgeld S. 210 ; im Zusammentreffen mit Betrug S. 211 .

$\S \S 47,73,74,146,147,148,149,150,151,152,246$, 263,360 Nr. 4 und 5 .

Ferner: Fälschung und wiederholte Verwendung von Stempel- und Postwertzeichen ( $\$ 275,276)$ mit Beispielen S. 215-218.

7. Fall: Der beleidigte Ratschreiber . . . . . . .

Lösung . . . . . . . . . . . . . . . . $219-23^{6}$

Allgemeiner Teil: Notwehr (allgemeine Erörterungen S. 233-236; der vorliegende Fall S. 231-232)

Besonderer Teil: Das Vergehen der Beleidigung (allgemeine Erörterungen) S. 219-228. Der vorliegende Fall: Üble Nachrede S. 228; Wahrnehmung berechtigter Interessen S. 229-230; Kompensation S. $230-232$; Publikationsbefugnis S. 233 .

$\S \S 53,61,65,18_{5}-200$.

8. Fall: Die unehelichen Kinder der Luise Baumeister .

Lösung . . . . . . . . . . . . . . . . $236-25^{\circ}$

Allgemeiner Teil: Mittelbare Täterschaft (allgemeine Erörterungen S. 237-241; der vorliegende Fall S. 245-246). Verbotsirrtum S. 243-244.

Besonderer Teil: Personenstandsdelikt (allgemeine Erörterungen S. 236-237; der vorliegende Fall S. 24I-246). Mittelbare (intellektuelle) Urkundenfälschung (allgemeine Erörterungen S. 246-249; der vorliegende Fall S. 249-250).

$\$ 4^{8}, 59,169,271,272$. 
9. Fall: Ein folgenschwerer Einfall . . . . . . . .

Lösung . . . . . . . . . . . . . $25^{\mathrm{I}-28} 3$

Allgemeiner Teil: Mittäterschaft (allgemeine Erorierungen S. 264-266; der vorliegende Fall S. 266). Aufforderung zum Verbrechen und deren Annahme (allgemeine Erörterungen S. 267; der vorliegende Fall S. 267-268). Anstiftung (allgemeine Erörterungen S. 268-272; der vorliegende Fall S. 272 -274). SicherungsmaBregeln, insbesondere Sicherungsverwahrung (allgemeine Erörterungen S. 275 bis 28o; der vorliegende Fall S. $280-28 \mathrm{I}$ ).

Besonderer Teil: Erpressung (allgemeine Erörterungen S. 25I-254; der vorliegende Fall S. 254). Räuberische Erpressung S. 255-256. Besonders schwerer Raub S. 257. Körperverletzung mit tödlichem Ausgang S. 257. Fahrlässige Tötung S. 257. Unterschlagung (an Gegenständen einer Leich $\epsilon$ ) S. 258. Brandstiftung (allgemeine Erörterunger $S$. $259-26$ I ; der vorliegende Fall S. 26 I-263). Tötungsdelikt S. 263. Unterlassene Anzeige (allgemeine Erörterungen S. 274-275; der vorliegende Fall S. 275). Der Raubmord S. $281-283$.

$\S \S 20 \mathrm{a}, 42 \mathrm{a}, 42 \mathrm{e}, 43,46,47,48,49 \mathrm{a}, 3^{8}$, I $39,21 \mathrm{I}$, $212,222,226,246,249,250,251,253,255,303,305$, 3о6, 3 го, 3 гоа.

10. Fall: Der weibliche Trunkenbold. . . . . . . .

Lösung . . . . . . . . . . . . . . . . . . 283-304

Allgemeiner Teil: Kausalzusammenhang (allgemeine Erörterungen S. $287-292$; der vorliegende Fall S. 292-293). Fahrlässigkeit (allgemeine Erörterungen) S. 293-295. Unterlassungsdelikte (allgemeine Erörterungen) S. 297-298. Eventualdolus S. $30 \mathrm{I}$.

Besonderer Teil: Giftbeibringung (allgemeine Erörterungen S. 283; der vorliegende Fall S. 284). Körperverletzung S. 284-285. Aussetzung (allgemeine Erörterungen S. 285; der vorliegende Fall S. 286-287, 292). Fahrlässige Tötung S. 295; im Zusammentreffen mit Aussetzung mit Todesfolge S. 295. Mord und Totschlag (allgemeine Erörterungen $\mathrm{S}$. 299-300; der vorliegende Fall S. 300-302). Unterlassene Hilfeleistung (allgemeine Erörterungen S. 302-303; der vorliegende Fall S. 303-304). $\$ \S$ I 38, I 39, 2 I I, 2 I 2, 22 I, 222, 223, 229, 330 . Ferner: Verkehrsflucht (Unfallflucht) S. 304 . 